Article

\title{
Plasticity through De-Twinning in Twinned BCC Nanowires
}

\author{
G. Sainath ${ }^{1, * \mathbb{D}}$, Sunil Goyal ${ }^{1,2}$ and A. Nagesha ${ }^{1,2}$ \\ 1 Materials Development and Technology Division, Indira Gandhi Centre for Atomic Research, Kalpakkam, \\ Tamilnadu 603102, India \\ 2 Homi Bhabha National Institute, Indira Gandhi Centre for Atomic Research, Kalpakkam, Tamilnadu 603102, India \\ * Correspondence: sg@igcar.gov.in or mohansainathan@gmail.com
}

Received: 18 March 2020; Accepted: 18 April 2020; Published: 1 May 2020

check for updates

\begin{abstract}
The deformation behaviour of twinned FCC nanowires has been extensively investigated in recent years. However, the same is not true for their BCC counterparts. Very few studies exist concerning the deformation behaviour of twinned BCC nanowires. In view of this, molecular dynamics (MD) simulations have been performed to understand the deformation mechanisms in twinned BCC Fe nanowires. The twin boundaries (TBs) were oriented parallel to the loading direction [110] and the number of TBs is varied from one to three. MD simulation results indicate that deformation under the compressive loading of twinned BCC Fe nanowires is dominated by a unique de-twinning mechanism involving the migration of a special twin-twin junction. This de-twinning mechanism results in the complete annihilation of pre-existing TBs along with reorientation of the nanowire. Further, it has been observed that the annihilation of pre-existing TBs has occurred through two different mechanisms, one without any resolved shear stress and other with finite and small resolved shear stress. The present study enhances our understanding of de-twinning in BCC nanowires.
\end{abstract}

Keywords: molecular dynamics simulations; BCC Fe nanowires; twin boundaries; de-twinning

\section{Introduction}

In recent years, twinned nanowires have attracted considerable attention for research due to their superior physical properties as compared to their perfect counterparts. Moreover, since twin boundaries (TBs) possess high symmetry and lowest interface energy, materials containing TBs exhibit better properties compared to those containing any other boundaries [1]. TBs can act both as barrier and carrier for dislocation motion, thus resulting in a simultaneous increase of strength and ductility [2]. TBs can also exhibit high thermal and mechanical stability [3], improve corrosion resistance, fracture toughness and strain rate sensitivity [4,5]. In view of this, the TBs are desirable planar defects in nanowires/nanocrystalline materials and many efforts have been made to synthesize and investigate the twinned nanowires and other nanocrystalline materials [6].

In order to understand the deformation behaviour of twinned FCC nanowires/nanopillars, many experimental and atomistic simulation studies have been carried out in the literature [2,6-9]. The results have shown that depending on the orientation of the TBs with respect to the loading direction, deformation mechanisms vary in nanowires and also many novel deformation mechanisms have been reported. For example, in nanopillars with TBs perpendicularly to the loading direction (transverse TBs), the dislocation-twin interactions like dislocation transmission, absorption, multiplication and stair-rod formations have been observed [2,6]. On the other hand, an extensive de-twinning is reported in nanopillars with slanted TBs [6]. The de-twinning is due to the easy glide of twinning partials along the TBs under finite shear stress. In addition to de-twinning, a novel pseudo-elasticity and shape memory effects have been discovered in nanowires with slanted TBs [10], 
which are distinct from those exhibited by the perfect nanowires [11]. The de-twinning mechanism has also been observed in nanowires with TBs parallel to the loading axis [12]. Unlike the nanowires with slanted TBs, the de-twinning in nanopillar containing axial TBs is surprising due to zero resolved shear stress on the pre-existing twin boundary. However, using experiments and atomistic simulations, Cheng et al. [12] have reported a de-twinning mechanism in nanopillars with axial twin boundary placed closed to the surface. This de-twinning resulted in a complete annihilation of the existing twin boundary, giving rise to defect-free nanopillar. The observation of unique de-twinning mechanism has been attributed to the migration of a junction consisting of twin boundary and other high angle grain boundary [12]. Similar annihilation of twin boundary due to de-twinning mechanisms has been observed during the bending of Ni nanowires with twin thickness less than $1 \mathrm{~nm}$ [13]. These observations clearly indicate that the de-twinning is quite common in twinned FCC nanowires with the exception of traverse twin boundaries.

However, all the above studies were focused on twinned FCC nanowires/nanopillars and little attention has been paid towards twinned BCC nanowires. There are only a couple of investigations in the literature on BCC nanowires containing twin boundaries $[14,15]$. These studies on BCC nanowires with transverse TBs have shown a strong tension-compression asymmetry in deformation mechanisms. With respect to axial TBs, Li et al. [16] have investigated the role of six-fold twin on the torsional deformation of BCC Fe nanowires. Except this, no study exists on the role of axial TBs in BCC nanopillars. Further, it is interesting to examine the possibility of the occurrence of de-twinning mechanism in twinned BCC nanowires. In view of this, the present study aimed at understanding the deformation mechanisms in axially twinned BCC Fe nanowires using molecular dynamics (MD) simulations. The results show an intersecting de-twinning mechanism involving the migration of twin-twin junction, which results in a complete annihilation of pre-existing TBs and also leads to the reorientation of the nanowire.

\section{MD Simulation Details}

The deformation behaviour of [110] BCC Fe nanowires with twin boundaries oriented parallel to the loading direction were considered in this study (Figure 1). The nanopillar is enclosed by [1-11] and [1-1-2] type side surfaces. The number of TBs is varied from one to three (Figure 1a-c). All the nanowires had a square cross section width $(d)=8.5 \mathrm{~nm}$ and length $(l)$ was twice the cross section width, i.e., $l=17 \mathrm{~nm}$. In order to mimic an infinitely long nanowire, periodic boundary conditions were chosen only along the wire axis, while the other directions were kept free. On these twinned nanowires, compression loading has been simulated using molecular dynamics (MD) simulations. MD simulations have been carried out in LAMMPS package [17] employing an embedded atom method (EAM) potential for BCC Fe given by Mendelev et al. [18]. The Mendelev EAM potential has been chosen mainly because several predictions obtained using this potential are in good agreement with experimental observations. For example, this potential correctly predicted deformation by twinning and dislocation slip based on nanopillar orientation in BCC Fe $[19,20]$, which is quite close to the experimental findings in ultra-thin BCC W nanopillars [21]. Before applying the compressive load, energy minimization has been performed by conjugate gradient method followed by an equilibration to a required temperature of $10 \mathrm{~K}$ in NVT ensemble with a Nose-Hoover thermostat. Velocity verlet algorithm has been used to integrate the equations of motion with a time step of 2 femto seconds. The compressive deformation was carried out at a constant strain rate of $1 \times 10^{8} \mathrm{~s}^{-1}$ with respect to initial box length. The visualization of TBs and dislocations is accomplished in AtomEye [22] and OVITO [23] packages using centro-symmetry parameter (CSP) and common neighbour analysis (CNA). 


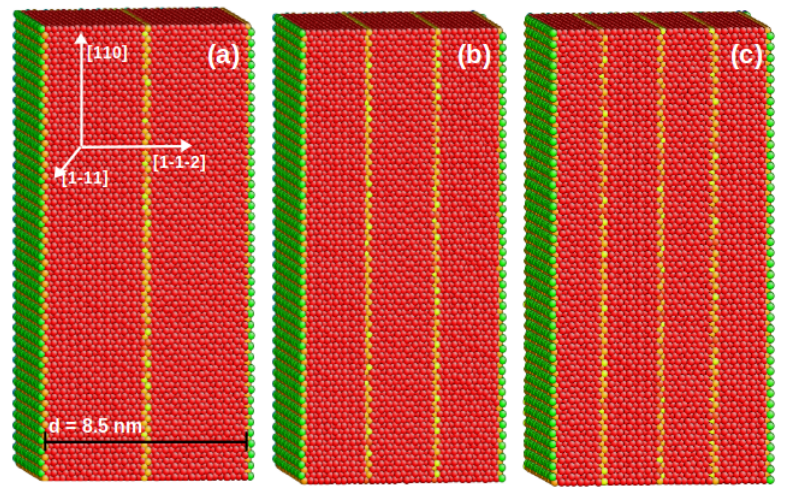

Figure 1. The initial configuration of BCC Fe nanowires containing (a) one, (b) two, and (c) three twin boundaries. The corresponding twin boundary spacings is of $4.25,2.83$ and $2.12 \mathrm{~nm}$, respectively. The nanowire orientation and cross-section width $(d)$ are indicated in $(\mathbf{a})$. The atoms are coloured according to their centro-symmetry parameter (CSP).

\section{Results And Discussion}

Figure 2a-h shows a typical deformation behaviour in axially twinned [110] BCC Fe nanowires containing two pre-existing twin boundaries. Similar behaviour has been observed in nanowires containing one and three TBs and therefore not shown here. It can be seen that the plastic deformation initiates by the nucleation of a twin from the nanowire corners (Figure 2a). With increasing deformation, the leading front of the twin approaches the existing twin boundary and penetrates into neighbouring grain (Figure 2b). Here, it is interesting to see that the twin penetration happens in two different ways; (1) it can penetrate directly to the next grain without any deviation in twinning plane and (2) it can pass onto a plane symmetrical to the original twinning plane (Figure 2b). Following the penetration, the leading twin front again passes through the second pre-existing twin boundary and results in the formation of twinned rhombohedron, which is enclosed by twin boundaries (Figure 2c). Also, the initially nucleated twin (Figure 2a) has grown significantly cutting across the pre-existing TBs (Figure 2c). It can be seen that the twin penetration and its growth has led to the annihilation and also the migration of pre-existing TBs (Figure 2c,d). With increasing strain, the twin growth dominates the deformation leading to the complete annihilation of pre-existing TBs (Figure 2e,f). Following the annihilation, the growing twin completely sweeps the nanowire length and leads to the reorientation of the nanowire from initial $<110>/\{111\}\{112\}$ orientation to $<001>/\{010\}\{310\}$ orientation (Figure $2 \mathrm{~g}, \mathrm{~h}$ ). Thus, in axially twinned BCC Fe nanowires there is an annihilation of pre-existing TBs. In other words, the twinned nanowires become twin free single crystalline nanowires with different orientation.

The deformation by twinning and reorientation in [110] twinned BCC Fe nanowires is similar to that observed in perfect [110] nanowires [19]. However, the annihilation of pre-existing TBs and the observation of reorientation in twinned nanowires is interesting. Similar to the present study, the annihilation of twin boundary due to de-twinning has also been observed recently in FCC nanowires [12]. However, this annihilation in FCC system has been observed only when the nanowire contains a single axial twin boundary located close to surface [12]. In the present study, the annihilation of TBs has been observed in nanowires containing one, two and three TBs irrespective of their location with respect to the surface. Further, there is an experimental evidence for the annihilation of twin boundaries due to de-twinning in FCC nanowires [12]. However, due to limited experimental studies on BCC nanowires compared to FCC, the annihilation of twin boundaries as a result of de-twinning has not been reported experimentally in BCC nanowires. Interestingly, a reversible twinning, which is also called de-twinning has been reported experimentally in BCC W nanowires [21]. Using in situ high-resolution transmission electron microscopy, it has been demonstrated that during loading, the $\mathrm{W}$ nanowire undergo deformation by twinning leading to twin growth. However, upon unloading, 
deformation proceeds by de-twinning, which is same as twinning, but in the reverse direction. As a result, the twin thickness is gradually reduced [21].
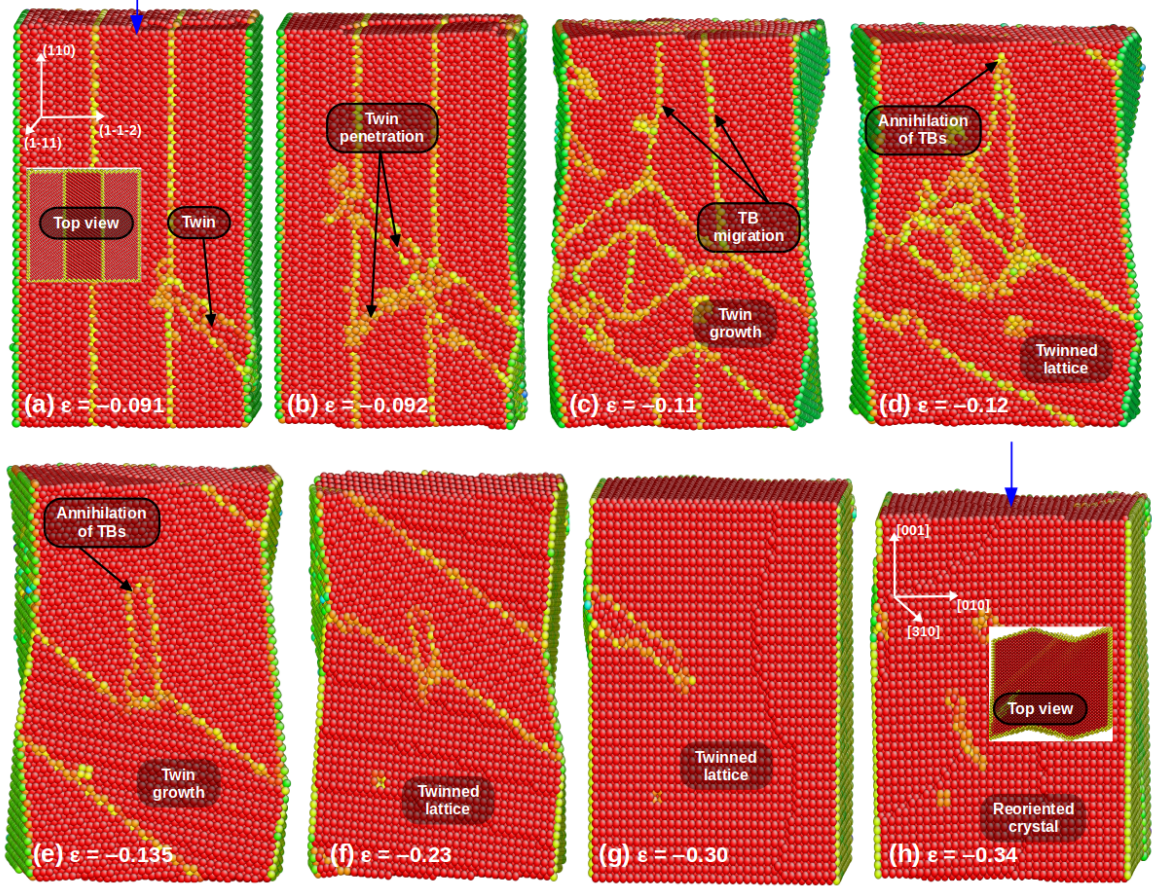

Figure 2. The atomic snapshots displaying the typical deformation behaviour in twinned BCC Fe nanowires at different strains; (a) twin nucleation, (b,c) twin penetration through existing TBs, (c,d) twin boundary migration and annihilation, $(\mathbf{d}-\mathbf{g})$ twin growth, and $(\mathbf{h})$ reoriented nanowire. The atoms are coloured according to their centro-symmetry parameter (CSP). The insets in (a,h) shows the top view of the nanowire.

The annihilation of pre-existing TBs in Figure 2a-h has occurred through two different mechanisms, one without any resolved shear stress (Figure 3) and other with finite and small resolved shear stress (Figure 4). Figure 3 shows a mechanism where a pre-existing twin boundary gets annihilated step by step due to the glide of $1 / 6<111>$ partial dislocations on newly formed TBs. The $1 / 6<111>$ partial dislocations nucleate from a twin-twin junction (Figure $3 b, f, j$ ) and glide on TBs (Figure $3 c-d, g-h, k-l$ ) whose migration results in twin growth. As a result of twin boundary migration and twin growth, the twin-twin junction moves step by step and leads to the annihilation of pre-existing twin boundary (Figure 3a,e,f). During this process, there is no apparent resolved shear stress on the pre-existing twin boundary as it is completely parallel to the loading axis (Figure 3a,e). It remains parallel till the partial dislocation activity is equal on both left and right TBs (Figure 3a-h). However, with increasing strain, due to the presence of some barriers, the partial dislocation activity on one twin boundary (left in this case) dominates over the other (Figure 3k,l). As a result, the pre-existing twin boundary slightly bends with respect to the loading axis (Figure 3i) which introduces a small resolved shear stress. Due to this shear stress, the partial dislocation activity also commences on the pre-existing twin boundary (Figure 4a,e). This partial dislocation activity further bends the twin boundary towards the other pre-existing twin boundary (Figure $4 \mathrm{~b}, \mathrm{c}, \mathrm{f}, \mathrm{g}$ ). As a result, they meet each other and annihilate over time (Figure $4 \mathrm{~d}, \mathrm{~h}$ ). This annihilation occurs under small resolved shear stress. Thus, two different mechanisms combinedly contribute to de-twinning in twinned BCC Fe nanowires (Figure 2). In contrast, the de-twinning in FCC nanowires occurs through a single mechanism of twin embryo nucleation followed by its expansion due to the migration of a special junction consisting of two TBs and one high angle grain boundary [12]. During this complete process, the existing twin 
boundary is always parallel to the loading axis, thereby getting annihilated under zero resolved shear stress [12].
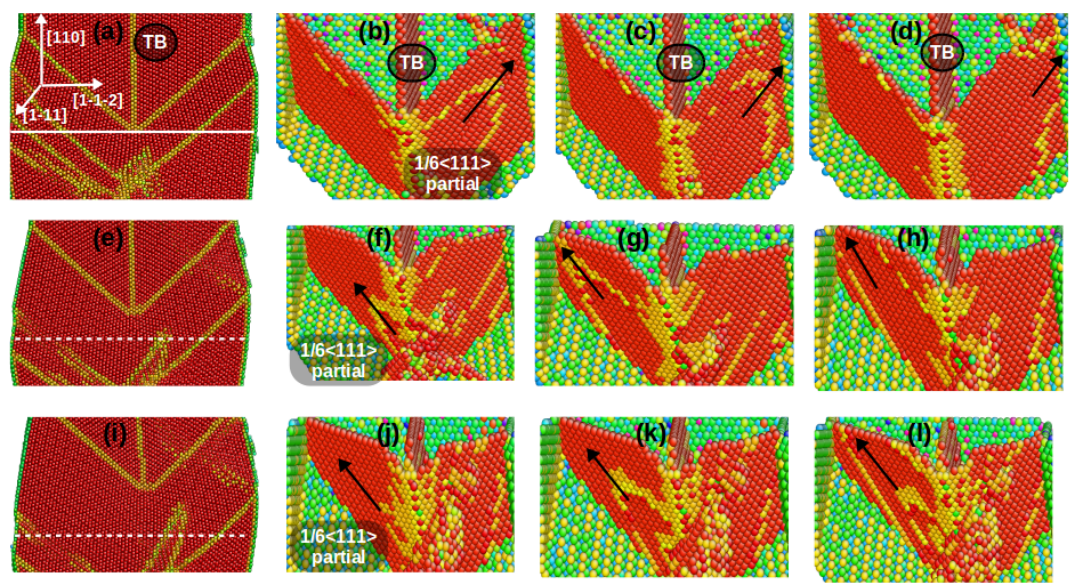

Figure 3. The annihilation of pre-existing twin boundary due to the step-by-step migration of the twin-twin junction $(\mathbf{a}, \mathbf{e}, \mathbf{i})$. The movement of twin-twin junction is aided by the continuous nucleation (from the twin-twin junction) $(\mathbf{b}, \mathbf{f}, \mathbf{j})$ and glide of $1 / 6<111>$ partial dislocations on newly formed twin boundaries $(\mathbf{c}, \mathbf{d}, \mathbf{g}-\mathbf{h}, \mathbf{k}, \mathbf{l})$. The atoms are coloured according to their centro-symmetry parameter (CSP). For clarity, the atoms in perfect BCC structure were removed in $(\mathbf{b}-\mathbf{d}),(\mathbf{f}-\mathbf{h})$ and $(\mathbf{j}-\mathbf{l})$.
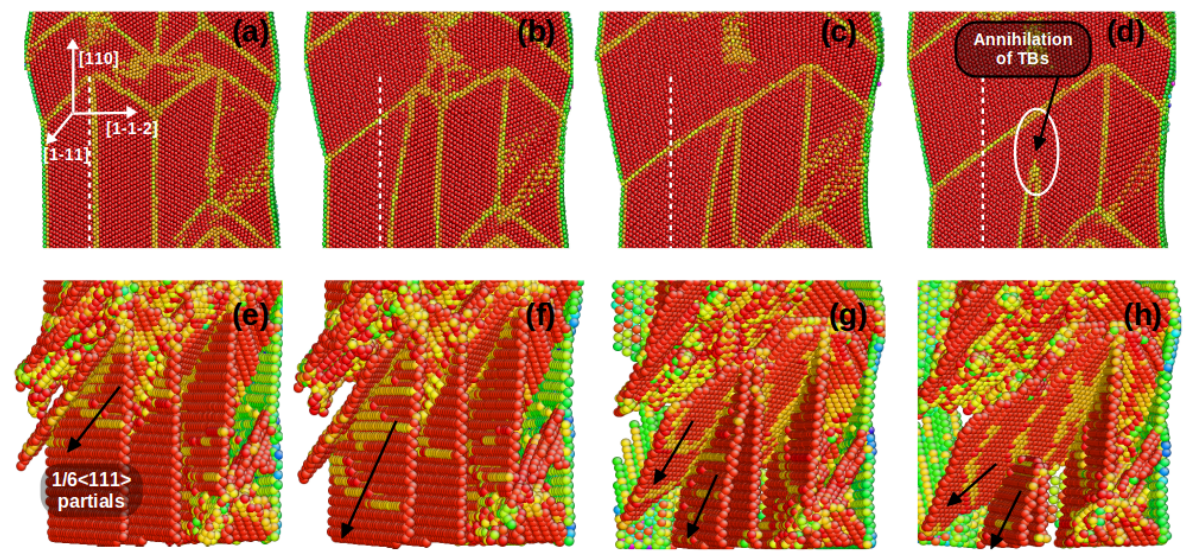

Figure 4. The annihilation of pre-existing twin boundary due to the bending and subsequent migration of pre-existing twin boundaries (a-d). This process is aided the by the continuous glide of $1 / 6<111>$ partial dislocations on the existing TBs. The atoms are coloured according to their centro-symmetry parameter (CSP). For clarity, the atoms in perfect BCC structure were removed in (e-h).

An investigation on the deformation behaviour of twinned nanowires also offers valuable insights into twin-twin interactions. Figure 5 shows two different types of twin-twin junctions observed in the present study. In one junction, three TBs meet at an angle of $120^{\circ}$ with respect each other as shown in Figure 5a (Y-junction), while in other junction, they meet at an angle of 60 and $240^{\circ}$ forming an arrow $(\downarrow)$ like junction (Figure $5 b$ ). Further, the annihilation of twin boundary with zero resolved shear stress has occurred mainly near arrow like twin-twin junctions (Figure 3), which participates more actively in de-twinning mechanisms. In the past, a junction containing six TBs (six-fold twin) has been inserted and studied for its evolution under torsion in $\alpha$-Fe [16]. However, no Y-junction or arrow like junctions have been observed. It is well known that in BCC systems, the TBs lie on $\{112\}$ planes and also three of the $\{112\}$ planes have the same $<111>$ zone axis as depicted in Figure $5 \mathrm{c}$. As a result of three $\{112\}$ planes having the same zone axis, the twin-twin junctions such as those shown in Figure 5a,b and also the six fold twins in Ref. [16] were feasible in BCC systems. In contrast, the six-fold 
twins were not compatible in FCC systems, where only up to five-fold twins were observed [24]. The arrangement of three $\{112\}$ planes as shown in Figure $5 \mathrm{c}$ is also responsible for the direct as well as symmetrical transmission of twin across the existing twin boundary as seen in Figure $2 b$. In a previous study [14], it has been shown that, like twins, dislocations can also either directly transmit through the twin boundary without any deviation in slip plane or they can transmit to symmetrical plane.
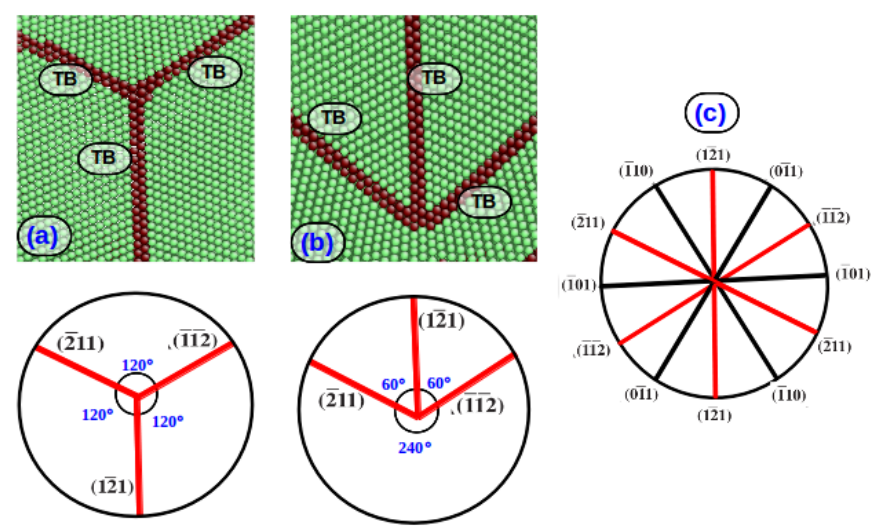

Figure 5. Two different twin-twin junctions observed in the present study; (a) Y-junction, where three TBs meet at an angle of $120^{\circ}$ with respect each other, $(\mathbf{b})$ arrow $(\downarrow)$ like junction, where three TBs meet at an angle of 60 and $240^{\circ}$, (c) the arrangement of $\{112\}$ slip planes, when viewed along $<111>$ direction. The atoms are coloured according to their common neighbour analysis (CNA).

\section{Conclusions}

Molecular dynamics simulations performed on the compressive loading of axially twinned [110] BCC Fe nanowire indicate that the deformation is dominated by a de-twinning mechanism involving the migration of a twin-twin junction. In the present study, two types of twin-twin junctions were observed. One is Y-junction where three TBs meet at an angle of $120^{\circ}$ with respect to each other and the other is an arrow $(\downarrow)$-like junction, where TBs meet at an angle of 60 and $240^{\circ}$. The results indicate that compared to the Y-junction, the arrow like junction participates more actively in de-twinning mechanisms. In all the nanowires, the de-twinning mechanism results in a complete annihilation of pre-existing TBs along with a change in nanowire orientation from initial $<110>$ to $<001>$. Further, it was observed that the annihilation of pre-existing TBs occurs through two different mechanisms, one without any resolved shear stress and other with a finite and small resolved shear stress. The annihilation of pre-existing TBs without any resolved shear stress has occurred through the step-by-step movement of the twin-twin junction due to the glide of $1 / 6<111>$ partial dislocations on newly formed twin boundaries. The annihilation of pre-existing TBs with finite shear stress occurred due to the slight bending of twin boundaries at higher strains. The present study significantly improves our understanding of de-twinning and also the stability of twin boundaries in BCC nanowires.

Author Contributions: G.S.: Conceptualization, Methodology, Data curation, Formal analysis and Writing-original draft; S.G.: Resources and Writing - review \& editing; A.N.: Resources and Writing-review \& editing. All authors have read and agreed to the published version of the manuscript.

Funding: This research received no external funding.

Conflicts of Interest: The authors declare no conflict of interest.

\section{References}

1. Lu, K.; Lu, L.; Suresh, S. Strengthening materials by engineering coherent internal boundaries at the nanoscale. Science 2019, 324, 349-352. [CrossRef]

2. Cao, A.J.; Wei, Y.G.; Mao, S.X. Deformation mechanisms of face-centered-cubic metal nanowires with twin boundaries. Appl. Phys. Lett. 2007, 90, 151909. [CrossRef] 
3. Wang, J.; Li, N.; Misra, A. Structure and stability of $\Sigma 3$ grain boundaries in face centered cubic metals. Philos. Mag. 2012, 93, 315-327. [CrossRef]

4. Singh, A.; Tang, L.; Dao, M.; Lu, L.; Suresh, S. Fracture toughness and fatigue crack growth characteristics of nanotwinned copper. Acta Mater. 2011, 59, 2437-2446. [CrossRef]

5. Deng, C.; Sansoz, F. Effects of twin and surface facet on strain-rate sensitivity of gold nanowires at different temperatures. Phys. Rev. B 2010, 81, 155430. [CrossRef]

6. Jang, D.; Li, X.; Gao, H.; Greer, J.R. Deformation mechanisms in nanotwinned metal nanopillars. Nat. Nanotechnol. 2012, 7, 594-601. [CrossRef]

7. Sainath, G.; Choudhary, B.K. Molecular dynamics simulation of twin boundary effect on deformation of $\mathrm{Cu}$ nanopillars. Phys. Lett. A 2015, 379, 1902-1905. [CrossRef]

8. Sun, J.; Li, C.; Han, J.; Shao, X.; Yang, X.; Liu, H.; Song, D.; Ma, A. Size effect and deformation mechanism in twinned Cu nanowires. Metals 2017, 7, 438. [CrossRef]

9. Rohith, P.; Sainath, G.; Sunil, G.; Nagesha, A.; Srinivasan, V.S. Role of axial twin boundaries on deformation dechanisms in Cu nanopillars. Philos. Mag. 2020, 100, 529-550. [CrossRef]

10. Deng, C.; Sansoz, F. A new form of pseudo-elasticity in small-scale nanotwinned gold. Extrem. Mech. Lett. 2016, 8, 201-207. [CrossRef]

11. Liang, W.; Zhou, M. Atomistic simulations reveal shape memory of fcc metal nanowires. Phys. Rev. B 2006, 73, 115409. [CrossRef]

12. Cheng, G.; Yin, S.; Chang, T.-H.; Richter, G.; Gao, H.; Zhu, Y. Anomalous tensile detwinning in twinned nanowires. Phys. Rev. Lett. 2017, 119, 256101. [CrossRef]

13. Wang, L.; Lu, Y.; Kong, D.; Xiao, L.; Sha, X.; Sun, J.; Zhang, Z.; Han, X. Dynamic and atomic-scale understanding of the twin thickness effect on dislocation nucleation and propagation activities by in situ bending of Ni nanowires. Acta Mater. 2015, 90, 194-203. [CrossRef]

14. Sainath, G.; Choudhary, B.K. Deformation behaviour of body centered cubic iron nanopillars containing coherent twin boundaries. Philos. Mag. 2016, 96, 3502-3523. [CrossRef]

15. Xu, S.; Startt, J.K.; Payne, T.G.; Deo, C.S.; McDowell, D.L. Size-dependent plastic deformation of twinned nanopillars in body-centered cubic tungsten. J. Appl. Phys. 2017, 121, 175101. [CrossRef]

16. Li, S.; Salje, E.K.H.; Jun, S.; Ding, X. Large recovery of six-fold twinned nanowires of $\alpha$-Fe. Acta Mater. 2017, 125, 296-302. [CrossRef]

17. Plimpton, S. Fast parallel algorithms for short-range molecular dynamics. J. Comp. Phy. 1995, 117, 1-19. [CrossRef]

18. Mendelev, M.I.; Han, S.; Srolovitz, D.J.; Ackland, G.J.; Sun, D.Y.; Asta, M. Development of new interatomic potentials appropriate for crystalline and liquid iron. Philos. Mag. 2003, 83, 3977-3994. [CrossRef]

19. Sainath, G.; Choudhary, B.K. Orientation dependent deformation behaviour of BCC iron nanowires. Comput. Mater. Sci. 2016, 111, 406-415. [CrossRef]

20. Dutta, A. Compressive deformation of Fe nanopillar at high strain rate: Modalities of dislocation dynamics. Acta Mater. 2017, 125, 219-230. [CrossRef]

21. Wang, J.; Zeng, Z.; Weinberger, C.R.; Zhang, Z.; Zhu, T.; Mao, S.X. In situ atomic-scale observation of twinning-dominated deformation in nanoscale body-centred cubic tungsten. Nat. Mater. 2015, 14, 594-600. [CrossRef] [PubMed]

22. Li, J. AtomEye: An efficient atomistic configuration viewer. Model. Simul. Mater. Sci. Eng. 2003, 11, 173. [CrossRef]

23. Stukowski, A. Visualization and analysis of atomistic simulation data with OVITO - the Open Visualization Tool. Modell. Simul. Mater. Sci. Eng. 2009, 18, 015012. [CrossRef]

24. Zhang, Z.; Huang, S.; Chen, L.; Zhu, Z.; Guo, D. Formation mechanism of fivefold deformation twins in a face centered cubic alloy. Sci. Rep. 2017, 7, 45405. [CrossRef] [PubMed]

(C) 2020 by the authors. Licensee MDPI, Basel, Switzerland. This article is an open access article distributed under the terms and conditions of the Creative Commons Attribution (CC BY) license (http:/ / creativecommons.org/licenses/by/4.0/). 